\title{
Procalcitonin in daily clinical practice: an evergreen tool also during a pandemic
}

\author{
Alessandro Russo ${ }^{1}$ (1) $\cdot$ Mario Venditti $^{1} \cdot$ Giancarlo Ceccarelli $^{1} \cdot$ Claudio Maria Mastroianni $^{1} \cdot$ Gabriella d'Ettorre $^{1}$
}

Received: 16 January 2021 / Accepted: 29 January 2021 / Published online: 12 February 2021

c) Società Italiana di Medicina Interna (SIMI) 2021

Procalcitonin (PCT) is widely considered an essential tool for identification and monitoring of patients with suspected and proved bacterial infections, to determine clinical severity and to assess the response to antibiotic therapy [1]. Moreover, recent studies demonstrated the association between PCT values and etiology of infection, with higher PCT concentrations detected in Gram-negative etiology, compared to Gram-positive and fungal infections [2,3].

In a recent real-world study on hospitalized patients admitted with respiratory symptoms and suspected lower respiratory tract infection (LRTI), Johnson et al. evaluated clinical outcomes and costs associated with PCT use in different clinical situations: patients with pneumonia, heart failure, viral respiratory infection, and chronic obstructive pulmonary disease [4]. Of importance, the PCT assessment was associated with reduced antibiotic use, length of stay, and mortality. Furthermore, patients who underwent PCT testing with a negative result were observed to have a 1.7-day shorter mean duration of antibiotics, a shorter mean length of stay of 1.5 days, and the lowest healthcare costs without an increase in adverse outcomes. Of interest, patients with PCT assessment, regardless of the PCT result, were less likely to be readmitted within 30 days.

As a matter of fact, it is plausible that the PCT concentrations can drive physicians to an early diagnosis and an appropriate choice of therapy, also avoiding readmissions for patients who may have received an incorrect empiric therapy. In the study of Johnson and coworkers, PCT testing results a valid tool to manage not only patients with bacterial LRTI but also those with other etiologies including noninfective and viral causes of hospitalization.

Alessandro Russo

alessandro.russo1982@gmail.com

1 Department of Public Health and Infectious Diseases, Policlinico "Umberto I", "Sapienza" University of Rome, P.le Aldo Moro 5, 00185 Rome, Italy
To date, PCT can be considered a useful tool for clinicians to manage at time of hospitalization patients with suspected infection by SARS-CoV-2 and to promptly distinguish this population from patients with bacterial etiology (see Fig. 1) [5]. Moreover, PCT results as one of the most important determinant to early detect and to monitor bacterial coinfections and superinfections in COVID-19 patients [5, 6]. Of importance, in a recent experience on 1461 patients with COVID-19, a PCT value $>0.5 \mathrm{ng} / \mathrm{mL}$ increased the risk of in-hospital mortality [7]. Finally, in a meta-analysis about risk factors for severity and death during COVID-19, a PCT value $>0.5 \mathrm{ng} / \mathrm{mL}$ was associated with a higher risk of progression to critical illness [8].

In vitro and in vivo models showed that PCT synthesis is stimulated by IL- 6 and TNF $\alpha$ increase [9]. The inflammatory responses induced in immune cells (but also epithelial and endothelial cells) were demonstrated to be crucial in stimulating a cytokine storm, leading to severe injury also in COVID-19 patients [10]. IL-6 is considered to be one of the key mediator of this cytokine storm, causing lung injury and the progression of COVID-19. As reported in several studies, the levels of serum IL-6 were elevated and IL-6 receptors were significantly expressed in patients affected by SARS-CoV-2 infection [11,12]. Thus, high PCT values may be associated with severity of SARS-CoV-2 infection and the measurement of PCT, especially in the first days of hospitalization, may help physicians to assess the hyper inflammatory activity and to rule out bacterial and fungal coinfection or superinfection [13, 14].

However, research is needed to clarify if the increase in serum inflammatory markers is directly caused by SARS$\mathrm{CoV}-2$ or should be considered an indirect consequence of patients' clinical status, especially in a population with chronic diseases that, like infectious diseases, can trigger a chronic proinflammatory state. Patients with such comorbidities are more likely to develop severe COVID-19 than healthy patients, at least partly for a dysfunction of innate immune response, increasing the risk of COVID-19 
Fig. 1 Management of COVID19 patients at emergency department and during hospitalization

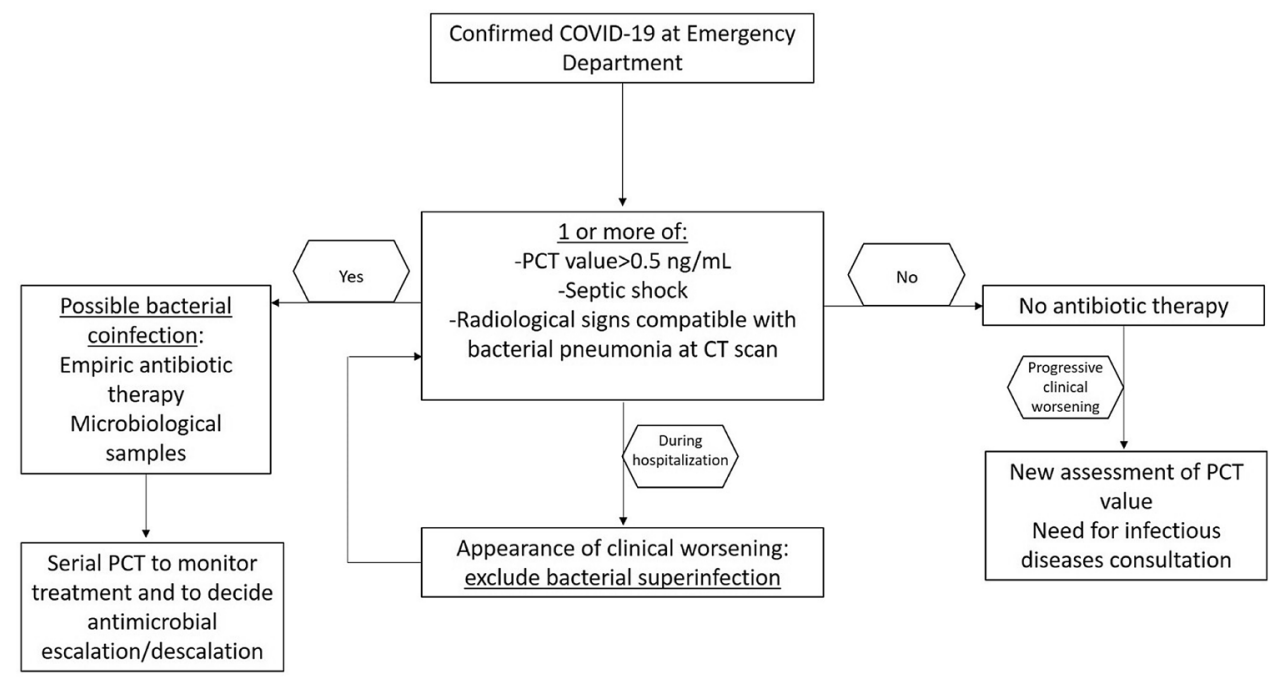

progression. Monitoring inflammatory markers may represent as an early warning system for progression to a more severe clinical condition.

It is important to underline that monitoring PCT levels can allow early detection of bacterial infections, which may reduce inappropriate prescription of antibiotics or trigger an early antibiotic therapy to treat the first stage of sepsis and other severe infective conditions [15]. Of importance, all the observed advantages associated with PCT utilization in the study of Johnson et al. were limited to patients not requiring intensive care unit (ICU) admission [4]. In this context, the use of PCT resulted to be more efficacious in ICU patients where the decrease of PCT of $>80 \%$ over $72 \mathrm{~h}$ from ICU admission may provide prognostic information in critically ill patients and drive physicians to the discontinuation of antibiotic therapy [16-18].

In conclusion, the high PCT values reported in COVID19 patients could be associated with the severity of infection and not only with the presence of bacterial coinfection or superinfection; for all these reasons, the combination of patient's clinical status with laboratory tests and imaging is crucial in daily practice to assess the likelihood of bacterial coinfection in patients with COVID-19 [19], (see Fig. 1). PCT should be included in a diagnostic stewardship to apply the well-known strategies for an appropriate antimicrobial prescription [20].

\section{Compliance with ethical standards}

Conflict of interest The authors declare that they have no conflict of interest.

Human and animal rights Not applicable.
Informed consent Not applicable.

\section{References}

1. Hamade B, Huang DT (2020) Procalcitonin: where are we now? Crit Care Clin 36:23-40

2. Bassetti M, Russo A, Righi E, Dolso E, Merelli M et al (2019) Role of procalcitonin in bacteremic patients and its potential use in predicting infection etiology. Expert Rev Anti Infect Ther 17:99-105

3. Bassetti M, Russo A, Righi E, Dolso E, Merelli M et al (2020) Role of procalcitonin in predicting etiology in bacteremic patients: report from a large single-center experience. J Infect Public Health 13:40-45

4. Johnson SA, Rupp AB, Rupp KL et al (2021) Clinical outcomes and costs associated with procalcitonin utilization in hospitalized patients with pneumonia, heart failure, viral respiratory infection, or chronic obstructive pulmonary disease. Intern Emerg Med. https://doi.org/10.1007/s11739-020-02618-3

5. Russo A, Bellelli V, Ceccarelli G, Marincola Cattaneo F, Bianchi L, Pierro R, Russo R, Steffanina A, Pugliese F, Mastroianni CM, d'Ettorre G, Sabetta F (2020) Comparison between hospitalized patients affected or not by COVID-19 (RESILIENCY study). Clin Infect Dis 18:ciaa11745. https://doi.org/10.1093/cid/ciaa1 745 ([Epub ahead of print])

6. Garcia-Vidal C, Sanjuan G, Moreno-García E, Puerta-Alcalde P, Garcia-Pouton N et al (2021) (2020) Incidence of co-infections and superinfections in hospitalized patients with COVID-19: a retrospective cohort study. Clin Microbiol Infect 27:83-88

7. Bahl A, Van Baalen MN, Ortiz L, Chen NW, Todd C, Milad M, Yang A, Tang J, Nygren M, Qu L (2020) Early predictors of inhospital mortality in patients with COVID-19 in a large American cohort. Intern Emerg Med 15:1485-1499

8. Zheng Z, Peng F, Xu B, Zhao J, Liu H, Peng J, Li Q, Jiang C, Zhou Y, Liu S, Ye C, Zhang P, Xing Y, Guo H, Tang W (2020) Risk factors of critical and mortal COVID-19 cases: a systematic literature review and meta-analysis. J Infect 81:e16-e25

9. Nijsten MW, Olinga P, The TH et al (2000) Procalcitonin behaves as a fast responding acute phase protein in vivo and in vitro. Crit Care Med 28:458-461 
10. Azkur AK, Akdis M, Azkur D, Sokolowska M, Van De Veen $\mathrm{W}$, Bruggen $\mathrm{MC}$ et al (2020) Immune response to SARS-CoV-2 and mechanisms of immunopathological changes in COVID-19. Allergy 75:1564-1581

11. Liu BW, Li M, Zhou ZG, Guan X, Xiang YF (2020) Can we use interleukin-6 (IL-6) blockade for coronavirus disease 2019 (COVID-19) — induced cytokine release syndrome (CRS)? J Autoimmun 111:102452

12. Liu J, Li S, Liu J, Liang B, Wang X, Wang H et al (2020) Longitudinal characteristics of lymphocyte responses and cytokine profiles in the peripheral blood of SARS-CoV-2 infected patients. EBioMedicine 55:102763

13. Danwang C, Endomba FT, Nkeck JR, Wouna DLA, Robert A, Noubiap JJ (2020) A meta-analysis of potential biomarkers associated with severity of coronavirus disease 2019 (COVID-19). Biomark Res 8:37

14. Russo A, Tiseo G, Falcone M, Menichetti F (2020) Pulmonary aspergillosis: an evolving challenge for diagnosis and treatment. Infect Dis Ther 9:511-524

15. Ji P, Zhu J, Zhong Z, Li H, Pang J, Li B, Zhang J (2020) Association of elevated inflammatory markers and severe COVID-19: a meta-analysis. Medicine (Baltimore) 99:e23315

16. Schuetz P, Maurer P, Punjabi V, Desai A, Amin DN, Gluck E (2013) Procalcitonin decrease over 72 hours in US critical care units predicts fatal outcome in sepsis patients. Crit Care 17:R115
17. Alessandri F, Pugliese F, Angeletti S, Ciccozzi M, Russo A, Mastroianni CM, d'Ettorre G, Venditti M, Ceccarelli G (2020) Procalcitonin in the assessment of ventilator associated pneumonia: a systematic review. Adv Exp Med Biol. https://doi. org/10.1007/5584_2020_591 ([Epub ahead of print])

18. Bassetti M, Russo A, Righi E, Dolso E, Merelli M, Cannarsa N, D'Aurizio F, Sartor A, Curcio F (2018) Comparison between procalcitonin and C-reactive protein to predict blood culture results in ICU patients. Crit Care 22:252

19. Falcone M, Bassetti M, Tiseo G, Giordano C, Nencini E, Russo A, Graziano E, Tagliaferri E, Leonildi A, Barnini S, Farcomeni A, Menichetti F (2020) Time to appropriate antibiotic therapy is a predictor of outcome in patients with bloodstream infection caused by KPC-producing Klebsiella pneumoniae. Crit Care $24: 29$

20. Wirz Y, Meier MA, Bouadma L et al (2018) Effect of procalcitonin-guided antibiotic treatment on clinical outcomes in intensive care unit patients with infection and sepsis patients: a patient-level meta-analysis of randomized trials. Crit Care 22:191

Publisher's Note Springer Nature remains neutral with regard to jurisdictional claims in published maps and institutional affiliations. 